\title{
刈割后两种不同体型植物的补偿式样对比研究
}

\author{
雷抒情 1 王海洋 ${ }^{1,2 *} \quad$ 杜国祯 ${ }^{2}$ 潘声旺 ${ }^{1}$ \\ （1 西南农业大学园艺园林学院, 重庆 400716）（2 兰州大学干早农业生态教育部重点实验室,兰州 730000）
}

\begin{abstract}
摘 要 对比了两种不同体型植物燕麦 (Avena sativa) 和油菜 (Brassica campestris) 在不同施肥水平下的刈割反应特 点。结果表明: 对于燕麦而言, 在不施肥条件下, 3 个时期的轻度刈割处理与对照相比, 其生物量、总生物量、果重、 果数等都有增加, 但只有某些指标出现超补偿; 在施肥条件下, 各种刚割处理后均没有发生超补偿。并且无论施肥 与否, 分菜期与拔节期的补偿指数均高于抽穗期的补偿指数。可以认为, 不施肥条件下营养期轻度刈割处理较有 利于燕麦的补偿生长。对于油菜而言, 花蕾期轻度刈割处理后植物补偿指数最大, 且施肥条件下的补偿指数高于 不施肥条件下的补偿指数。比较两种植物在不同资源下补偿反应的特点, 可认为因休眠芽位置及其活动方式不同 而所造成的体型差异对植物的补偿反应式样有很大影响。
\end{abstract}

关键词 刏割 补偿 休眠芽 体型

\section{COMPENSATORY GROWTH RESPONSES OF TWO PLANTS WITH DIFFERENT GROWTH FORMS AFTER CLIPPING}

\author{
LEI Shu-Qing ${ }^{1}$ WANG Hai-Yang ${ }^{1,2 *}$ DU Guo-Zhen ${ }^{2}$ and PAN Sheng-Wang ${ }^{1}$ \\ (1 College of Horticulture and Landscape, Southwest Agricultural University, Chongqing 400716, China) \\ (2 Key Laboratory of Arid Agroecology of Ministry of Education, Lanzhou University, Lanzhou 730000, China)
}

\begin{abstract}
Identifying mechanisms of tolerance to herbivore damage will facilitate attempts to understand the role of tolerance in the evolutionary and ecological dynamics of plants and herbivores. Several external factors, such as water availability, nutrient availability, intensity of damage, and timing of damage, will affect the ability of individual plants to tolerate damage by mediating internal mechanisms. Though interspecific comparisons are useful for identifying possible mechanisms, direct comparisons between tolerance and putative mechanism have been made almost exclusively in interspecies or interpopulation studies.

This study compared the compensatory responses of Avena sativa and Brassica campestris, which belong to different growth forms, to clipping under two fertilization treatments. The results showed that, for Avena sati$v a$, under no fertilization treatment, the biomass, total biomass, fruit weight and number of fruits were greater than in the control, but only some aspects resulted in overcompensation. Under fertilized conditions, clipping treatments did not cause any overcompensation. Whether fertilized or not, the index of compensation during the tillering stage and jointing stage were both higher than during the flowering stage. Thus, under the no fertilization treatment, the low clipping treatment during the vegetative stage was shown to benefit Avena sativa. With respect to Brassica campestris, the index of compensation was greatest in the low clipping treatment during the flower bud stage, and was enhanced under fertilization. These results indicate that clipping during the reproductive stage can help compensatory growth in Brassica campestris. The different responses to clipping were attributed to the different growth forms, which had different positions and activities of dormant buds.
\end{abstract}

Key words Clipping, Compensation, Dormant bud, Growth form

补偿生长是植物具有的一种普遍现象 (Belsky, 1986)。植物补偿反应式样与伤害发生的时间、强 度、频度以及土壤的资源状况有关 (Belsky et al., 1993; Rosenthal \& Kotanen, 1994)。一般认为在高资 源情况下植物的补偿效果好 (Maschinski \& Whitham,
1989), 但是也存在相反的观点 ( Hilbert et al., 1981)。植物在个体水平上的补偿机制一般包括如 下 4 个方面: 1 ) 被食草动物采食后, 剩余叶片光合作 用增强，在一段时间内可以积累更多的同化物质 (Trumble et al. ，1993);2)植物伧藏资源有一个再分 
配过程, 这种分配是以牺牲其它器官的生理和代谢 为代价的 (Evans, 1991); 3 ) 剩余组织的相对生长速 率增大, 因而在有限的生长季节内可能产生超补偿 (Hilbert et al.，1981); 4)伤害解除顶端优势, 刺激休 眠芽的活动, 使植物产生较多的分枝结构 (Paige \& Whitham, 1987; Lennartsson et al ., 1998)。

Tuomi 等 (1994)认为, 植物的补偿程度与植食者 的采食强度以及休眠芽的多少有关。休眠芽相对较 少的情况下,轻度伤害后超补偿才能产生。休眠芽 较多的情况下, 重度伤害后超补偿可能产生。那么 据此理论, 植物休眠芽位置及其活动方式不同而造 成体型差异的植物也应该具有不同补偿反应形式。 迄今为止, 比较不同体型植物在不同资源水平下的 补偿反应方面的研究还很少见有报道。本文选择了 基部分菜的燕麦 (Avena sativa) 与上部分枝的油菜 (Brassica campestris) 作为试验材料, 探讨不同植物的 补偿反应差异是否与休眠芽位置和活动有关。

\section{1 材料和方法}

\section{1 试验地点与试验方法}

试验地点位于甘肃省甘南藏族自治州合作市兰 州大学干旱农业生态教育部重点实验室高寒草甸生 态系统定位站, 地理位置为 $34^{\circ} 55^{\prime} \mathrm{N}, 102^{\circ} 53^{\prime} \mathrm{E}$, 海 拔 2900 m。自然条件已有报道(王海洋等, 2003)。

2003 年 5 月, 在 $300 \mathrm{~m}^{2}$ 的实验地进行施肥处 理。一组不施肥, 作为施肥对照区; 另一组施磷酸二 铵(有效氮含量 $18 \%$, 有效磷含量 $46 \%$ ), 施肥标准 为 $60 \mathrm{~g} \cdot \mathrm{m}^{-2}$ 。增施磷肥的原因是有证据表明磷肥 可以提高人工草场的使用寿命 (杜国祯和王刚, 1995）。5 月 20 日分别播种燕麦和油菜。播种密度 为 400 粒 $\cdot \mathrm{m}^{-2}$, 幼苗密度控制在 200 株 $\cdot \mathrm{m}^{-2}$ 。当燕 麦进入分葢期, 油菜 3 片叶子时分别选择高度、分莧 数和叶片数相近的植株挂牌定株。每种组合的刈割 处理定株 160 个。油菜、燕麦共计 5760 株。其中, 在每个处理的定株中, 60 个用于生物量、果实数等 收获指标的统计, 24 个(刏割前后, 每次 12 样株)用 于两次光合速率的测定, 12 个用于特定叶面积干重 $(S L A)$ 测定, 40 个样株用于同化产物再分配的测定， 其它样株作为预留。

刚割试验设计:在施肥区和施肥对照区, 于燕麦 分藍期、拔节期和抽穗期, 油菜营养期、花蕾期和开 花期, 模拟自然放牧条件, 按留茬高度分别进行不同 强度的刈割处理。共计有 7 种刚割组合处理: 1$)$ 燕 麦分藥期、油菜营养期轻度刈割, 刈割部分生物量占
地上部分生物量的 $10 \%$ 左右;2)燕麦分睬期、油菜 营养期重度刈割, 刏割部分生物量占地上部分生物 量的 $60 \%$ 左右。由于这个时期燕麦、油菜体型较 小, 刏叶处理未破坏分生组织; 3) 燕麦拔节期、油菜 花蕾期轻度刚割, 刈割强度同 1);4)燕麦拔节期、油 菜花蕾期重度刈割, 刏割强度同 2);5)燕麦抽穗期、 油菜开花期轻度刚割, 刏割强渡同 1);6)燕麦抽穗 期、油菜开花期重度刚割, 刈割强度同 2);7）对照不 刈割 CK。

刏割处理的同时, 以相同的强度刈割定株周围 的植株, 以减少植株对光的竞争。定株刏割的部分 装袋, 带回实验室。8 月 27 日以个体为单位分地 上、地下收获燕麦和油菜, 地上部分测定植株的高 度、分枝数、总分藍数、有效分藮数、果实数和茎、叶、 果实、现存生物量、总生物量, 地下部分测定生物量。 所有生物量均在 $70{ }^{\circ} \mathrm{C}$ 下 $48 \mathrm{~h}$ 烘干至恒重。在电子 天平 (Sartorius BP 190S Max $200 \mathrm{~g} \mathrm{~d}=0.1 \mathrm{mg}$ ) 上称 重。数据在 STATISTICA5.0 统计软件上做方差分析 (ANOVA)。

\section{2 补偿反应式样的判断}

刏割植株与对照植株干重或种子数的比值作为 刏割后植物的反应指标, 定义为补偿指数 (Compensation index, $C I$ )（王海洋等, 2003)。分别计算被刚 割植株果实数、果实重、生物量(现存生物量)、总生 物量 ( 刈割部分 + 现存生物量) 与对照植株指标之间 的比值。根据补偿指数, 结合 ANOVA 检验结果判 断补偿反应式样, 若 $C I$ 大于 1 , 且则割处理植株的 指标与对照植株之间存在显著差异, 则为超补偿; 若 $C I$ 值稍大于, 等于或稍小于 1 , 且方差分析不显著, 则为等量补偿; 若 $C I$ 小于 1 , 且方差分析显著, 则判 断为不足补偿。

\section{2 结果与分析}

\section{1 刈割前植株的状况}

不施肥条件下燕麦 3 个时期植株的平均高度分 别为 $(21.6 \pm 0.32) 、(27.0 \pm 0.38)$ 和 $(46.0 \pm 0.40)$ $\mathrm{cm}$ 。平均叶片数为 $(2.41 \pm 0.08) 、(2.75 \pm 0.10)$ 和 (2.69 \pm 0.16$)$ 个。各个刚割处理之间基本相似 $(d f$ $=119, p>0.05)$; 施肥条件下, 植株的平均高度分 别为 $(24.1 \pm 0.41) 、(41.0 \pm 0.49)$ 和 $(66.6 \pm 1.07)$ $\mathrm{cm}$, 平均叶片数为 $(3.82 \pm 0.10) 、(4.93 \pm 0.16)$ 和 $(5.05 \pm 0.22)$ 个, 刚割处理之间没有显著差异 $(d f=$ $119, p>0.05$ )。不施肥条件下, 油菜 3 个时期的平 均高度分别为 $(8.7 \pm 0.18) 、(22.3 \pm 0.59)$ 和 $(34.1 \pm$ 
$0.69) \mathrm{cm}$, 平均叶片数为 $(4.3 \pm 0.09) 、(4.7 \pm 0.04)$ 和 $(4.3 \pm 0.03)$ 个。刚割处理之间无显著差异 $(d f=$ $119, p>0.05)$ 。施肥条件下, 油菜 3 个时期的平均 高度分别为 $(10.2 \pm 0.23) 、(33.9 \pm 0.57) 、(63.1 \pm$ $1.21) \mathrm{cm}$ 平均叶数分别为 $(5.9 \pm 0.07) 、(4.8 \pm$ $0.05) 、(4.6 \pm 0.03)$ 个。 3 个时期刚割处理之间无显 著差异 $(d f=119, p>0.05)$ 。因此, 油菜、燕麦各个 处理时期定株大小基本一致。

2.2 不同资源水平下, 不同时期不同强度的刈割处
理对燕麦的补偿影响

不同资源水平下, 不同时期不同强度的刈割处 理下燕麦的反应见图 1 和表 1 。不施肥条件下, 分 薯期、拔节期的轻度刈割处理均使果重、果数、生物 量、总生物量与对照相比有显著增加 $(p<0.001, d f$ $=119,1.42<C I<1.77)$ 。抽穗期的轻度刈割处理 虽然使果实数、总生物量上与对照相比显著增加 $(p$ $<0.05, d f=119,1.23<C I<1.37)$ 。但是果重、生 物量有等量补偿的特点 $(0.98<C I<1.19)$ 。分菜期
不施肥 Unfertilized
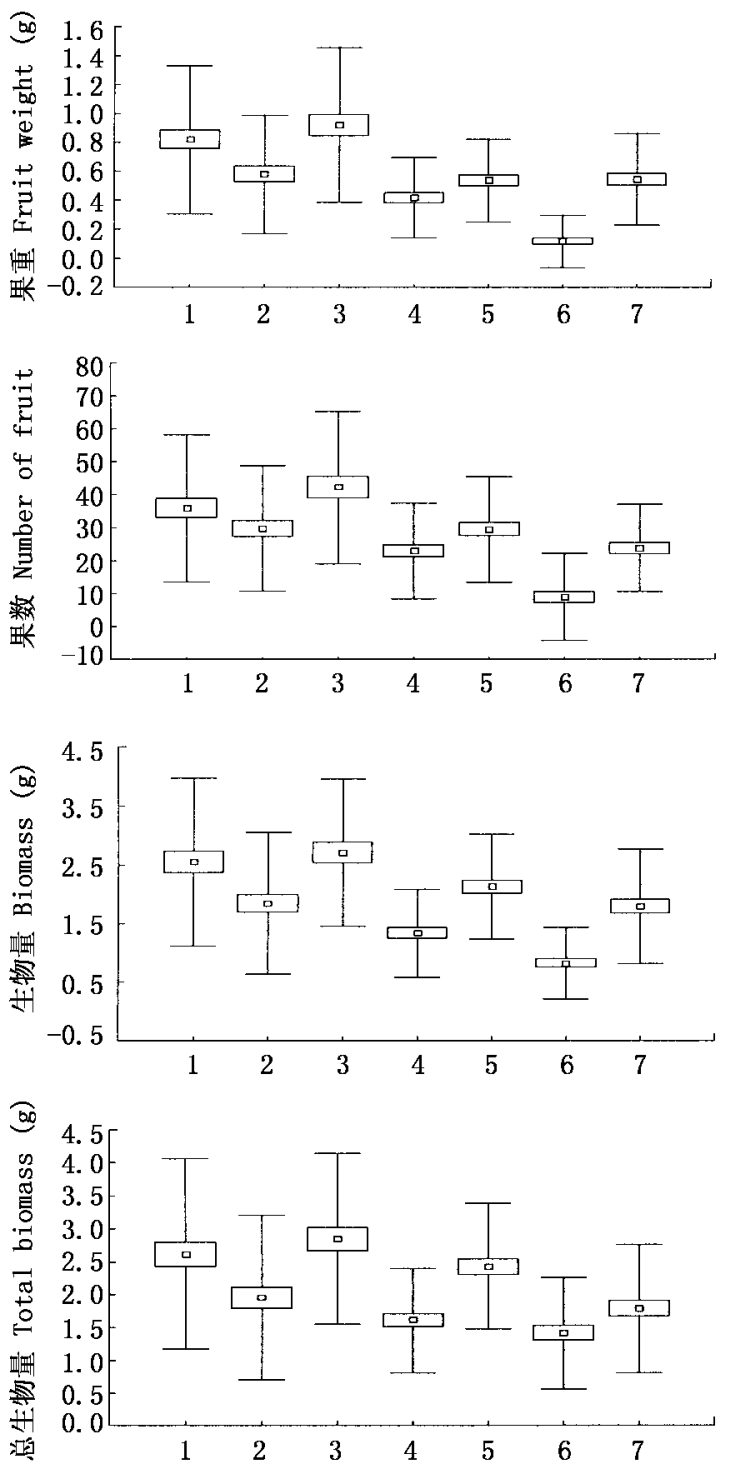

施肥 Fertilized
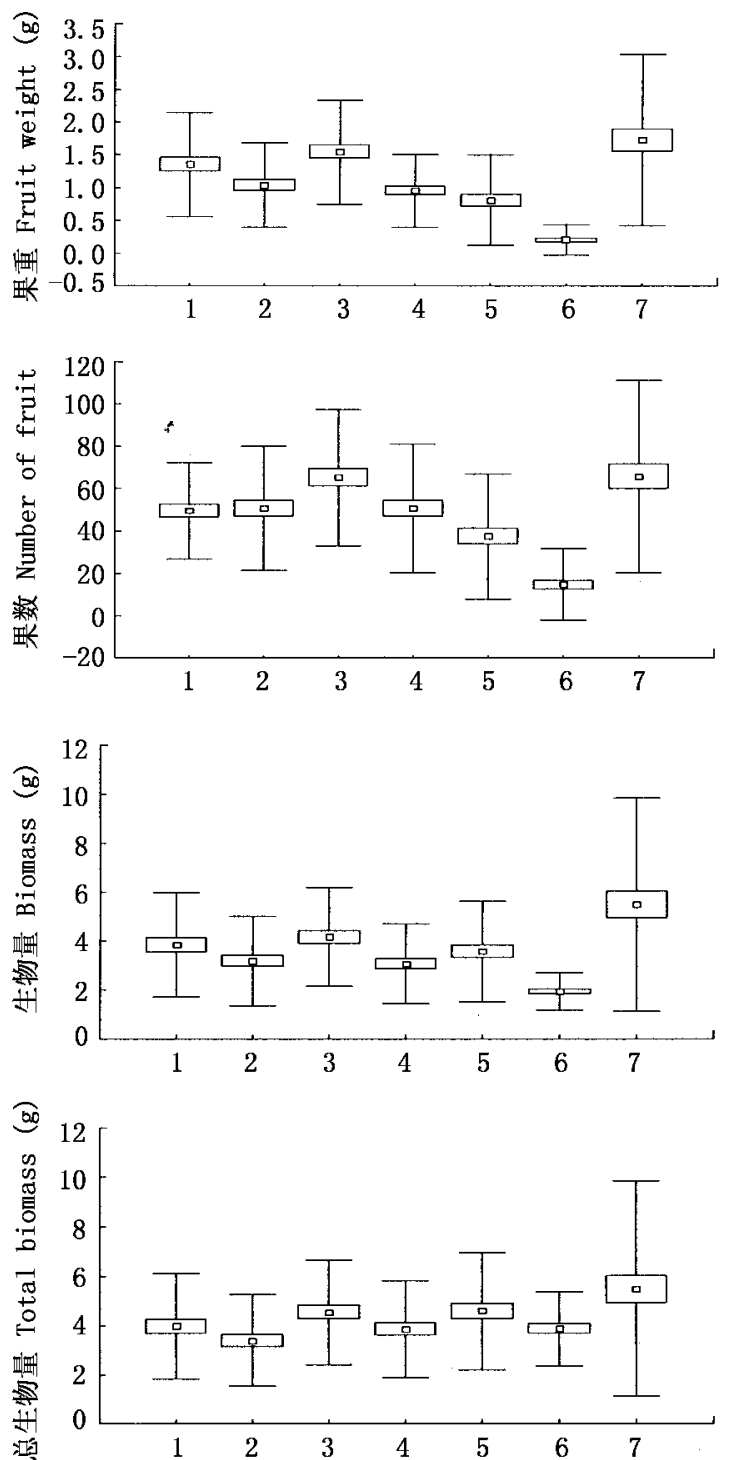

图 1 刈割时间、刏割强度及施肥处理对燕麦生长和生殖的影响

Fig. 1 The effect of clipping time, clipping intensity and fertilization on growth and reproduction of Avena sativa

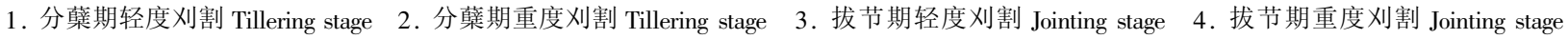
high 5. 抽穗期轻度刈割 Flowering stage 6. 抽穗期重度刈割 Flowering stage 7. 对照 CK 
表 1 燕麦施肥和不施肥条件下不同刈割处理与对照之间植物各指标的补偿指数

Table 1 The compensation index of Avena sativa between control plot (CK) and treatment clipped in different time and intensity under two levels of fertilization

\begin{tabular}{|c|c|c|c|c|c|}
\hline \multirow{2}{*}{$\begin{array}{c}\text { 处理 } \\
\text { Treatment }\end{array}$} & \multirow{2}{*}{$\begin{array}{c}\text { 刈割/对照 } \\
\text { Clipping/Control }\end{array}$} & \multicolumn{4}{|c|}{ 补偿指数 Compensation index } \\
\hline & & 果重 Fruit weight & 果数 No. of fruit & 生物量 Biomass & 总生物量 Total biomass \\
\hline 不施肥 & $1 / \mathrm{CK}$ & $1.500^{* *}$ & $1.502^{* *}$ & $1.426^{* *}$ & $1.464^{* *}$ \\
\hline \multirow[t]{6}{*}{ Unfertilized } & $2 / \mathrm{CK}$ & 1.060 & 1.247 & 1.031 & 1.094 \\
\hline & 3/CK & $1.689^{* *}$ & $1.766^{* *}$ & $1.515^{* *}$ & $1.590^{* *}$ \\
\hline & 4/CK & $0.764^{*}$ & 0.963 & $0.745^{* *}$ & 0.900 \\
\hline & $5 / \mathrm{CK}$ & 0.981 & $1.236^{*}$ & 1.188 & $1.361^{* *}$ \\
\hline & 6/CK & $0.213^{* *}$ & $0.374^{* *}$ & $0.460^{* *}$ & $0.791^{*}$ \\
\hline & $\mathrm{CK} / \mathrm{CK}$ & 1.000 & 1.000 & 1.000 & 1.000 \\
\hline 施肥 & 1/CK & 0.783 & $0.753^{*}$ & $0.701^{*}$ & $0.725^{*}$ \\
\hline \multirow[t]{6}{*}{ Fertilized } & $2 / \mathrm{CK}$ & $0.601^{* *}$ & $0.771^{*}$ & $0.579^{* *}$ & $0.620^{* *}$ \\
\hline & $3 / \mathrm{CK}$ & 0.894 & 0.990 & $0.761^{*}$ & 0.828 \\
\hline & $4 / \mathrm{CK}$ & $0.551^{* *}$ & $0.771^{*}$ & $0.560^{* *}$ & $0.704^{* *}$ \\
\hline & $5 / \mathrm{CK}$ & $0.468^{* *}$ & $0.569^{* *}$ & $0.651^{*}$ & 0.836 \\
\hline & 6/CK & $0.118^{* *}$ & $0.225^{* *}$ & $0.355^{* *}$ & $0.707^{* *}$ \\
\hline & $\mathrm{CK} / \mathrm{CK}$ & 1.000 & 1.000 & 1.000 & 1.000 \\
\hline
\end{tabular}

$* *: p<0.01 \quad *: p<0.05 \quad 1 、 2 、 3 、 4 、 5 、 6:$ 同图 1 See Fig. 1

的重度刈割与对照相比在上述 4 个指标上发生了等 量补偿 $(1.03<C I<1.25)$ 。拔节期的重度刚割处 理, 果实数、总生物量发生等量补偿 $(0.90<C I<$ $0.97)$ 。然而果实重、生物量发生不足补偿 $(p<$ $0.05, d f=119,0.74<C I<0.76)$ 。抽穗期的重度刈 割处理与对照相比较, 上述 4 个指标均发生不足补 偿 $(p<0.05, d f=119,0.21<C I<0.80)$ 说明, 营养 期的轻度刈割有利于燕麦的恢复补偿, 且补偿效果 在拔节期最好, 越是生长期后期, 补偿效果越差。表 明植物刚割后的补偿反应存在阈值, 超过此阈值的 刏割处理, 植物的补偿效果变差。3 个时期的重度
刚割处理与对照比较补偿效果均差。重度刚割不利 于燕麦的补偿生长。施肥条件下, 任何组合的刚割 处理与对照相比较上述 4 个指标的补偿效果都差, 补偿指数均小于 1.02 。说明, 施肥并没有提高燕麦 的补偿能力。

2.3 不同资源水平下, 不同时期、不同强度的刈割 处理对油菜的补偿影响

不同资源水平下,不同时期、不同强度的刈割处 理后油菜的反应见图 2 和表 2 。

对于油菜而言, 不施肥条件下, 营养期的轻度刈 割均使果重、果数、生物量、总生物量上发生了等量

表 2 施肥和不施肥条件下不同刚割处理与对照之间植物各指标的补偿指数

Table 2 The compensation index of Brassica campestris between control plot (CK) and treatment plant clipped in different time and intensity under two levels of fertilization

\begin{tabular}{|c|c|c|c|c|c|}
\hline \multirow{2}{*}{$\begin{array}{c}\text { 处理 } \\
\text { Treatment }\end{array}$} & \multirow{2}{*}{$\begin{array}{c}\text { 刚割/对照 } \\
\text { Clipping/Control }\end{array}$} & \multicolumn{4}{|c|}{ 补偿指数 Compensation index } \\
\hline & & 果重 Fruit weight & 果数 No. of fruit & 生物量 Biomass & 总生物量 Total biomass \\
\hline 不施肥 & $1 / \mathrm{CK}$ & 0.963 & 0.984 & 0.899 & 1.008 \\
\hline \multirow[t]{6}{*}{ Unfertilized } & $2 / \mathrm{CK}$ & $0.193^{* *}$ & $0.236^{* *}$ & $0.539^{* *}$ & $0.680^{*}$ \\
\hline & 3/CK & $0.506^{* *}$ & $0.779^{*}$ & 1.179 & $1.264^{*}$ \\
\hline & 4/CK & $0.240^{* *}$ & $0.528^{* *}$ & 0.858 & 1.034 \\
\hline & $5 / \mathrm{CK}$ & $0.364^{* *}$ & $0.444^{* *}$ & 0.836 & 0.939 \\
\hline & 6/CK & $0.096^{* *}$ & $0.086^{* *}$ & $0.616^{*}$ & 0.999 \\
\hline & $\mathrm{CK} / \mathrm{CK}$ & 1.000 & 1.000 & 1.000 & 1.000 \\
\hline 施肥 & $1 / \mathrm{CK}$ & $0.589^{* *}$ & $0.739^{* *}$ & $0.582^{* *}$ & $0.647^{* *}$ \\
\hline \multirow[t]{6}{*}{ Fertilized } & $2 / \mathrm{CK}$ & $0.223^{* *}$ & $0.280^{* *}$ & $0.371^{* *}$ & $0.450^{* *}$ \\
\hline & 3/CK & 1.165 & $1.444^{* *}$ & $1.392^{* *}$ & $1.431^{* *}$ \\
\hline & 4/CK & $0.320^{* *}$ & $0.431^{* *}$ & $0.596^{* *}$ & $0.694^{* *}$ \\
\hline & $5 / \mathrm{CK}$ & 1.089 & 1.240 & $1.306^{*}$ & $1.437^{* *}$ \\
\hline & 6/CK & $0.175^{* *}$ & $0.196^{* *}$ & $0.507^{* *}$ & $0.775^{*}$ \\
\hline & CK/CK & 1.000 & 1.000 & 1.000 & 1.000 \\
\hline
\end{tabular}



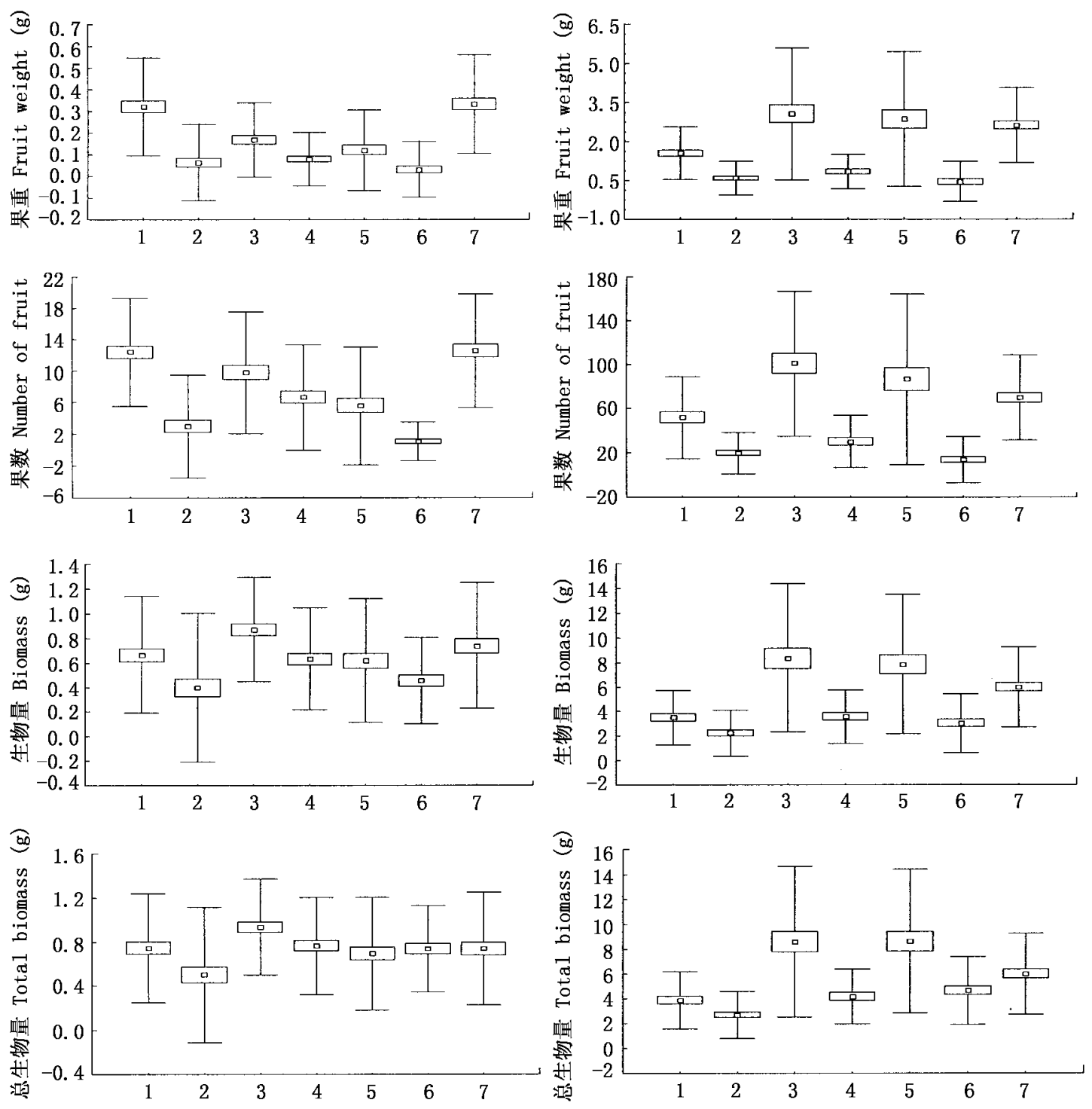

图 2 刈割时间、刈割强度及施肥处理对油菜生长和生殖的影响

Fig. 2 The effect of clipping time, clipping intensity and fertilization on growth and reproduction of Brassica campestris

1. 营养期轻度刚割 Vegetative stage 2. 营养期重度刚割 Vegetative stage 3. 花蕾期轻度刚割 Flower bud stage 4. 花蕾期重度刚割 Flower bud stage 5. 开花期轻度刈割 Flowering stage 6. 开花期重度刈割 Flowering stage 7. 对照 CK

补偿 $(0.89<C I<1.01)$ 。重度刚割上述 4 个指标都 产生了不足补偿 $(p<0.05, d f=119,0.19<C I<$ $0.68)$ 。花蕾期的轻度刚割总生物量发生了超补偿 $(p<0.05, d f=119, C I=1.264)$ 。重度刚割总生物 量等量补偿 $(C I=1.034)$ 。轻、重度刈割使现存生物 量有等量补偿特点 $(0.85<C I<1.18)$ 。果重、果数 则都发生了不足补偿 $(p<0.05, d f=119,0.24<C I$ $<0.78)$ 。开花期的轻、重度刈割总生物量有等量补 偿特点 $(0.93<C I<1.00)$ 。轻度刚割生物量等量补 偿 $C I=0.84$ 。重度刈割则为不足补偿 $(p<0.001$, $d f=119, C I=0.62)$ 。果数、果重均为不足补偿 $(p$ $<0.001, d f=119,0.08<C I<0.45)$ 。

施肥条件下, 营养期轻、重度刈割处理, 花蕾期 和开花期的重度刚割处理, 上述 4 个指标都是不足 补偿 $(p<0.05, d f=119, C I<0.78)$ 。生殖生长早 期的轻度刚割处理, 果重上等量补偿 $(C I=1.165)$ 。

果数、现存生物量、总生物量上为超补偿 $(p<$ $0.01, d f=119,1.391<C I<1.444)$ 。生殖生长的晚 期轻度刈割处理, 虽然生物量、总生物量产生了超补 偿 $(p<0.05, d f=119,1.305<C I<1.438)$ 。但是, 
果重、果数发生了等量补偿 $(1.088<C I<1.241)$ 。

结果说明, 生殖生长期的轻度刈割有利于油菜 的恢复生长, 补偿能力花蕾期达到最大, 并且施肥条 件下的补偿指数要高于不施肥条件下的补偿指数, 施肥提高了油菜对伤害的补偿能力。

\section{3 结论与讨论}

影响植物补偿反应的外部因素一般包括伤害时 间、伤害强度、土壤的资源状况等。Lennartsson 等 (1998)提出, 植物受伤害后的补偿反应程度与伤害 发生的时间密切相关, 伤害诱导的超补偿反应仅发 生在特定的诱导期 (Inductive time period, ITP)。本 试验中燕麦在拔节期、油菜在花蕾期的补偿效果最 高, 与该结论一致。但是, 导致燕麦在营养期、油菜 在生殖期补偿反应不一致的差异可能是休眠芽所处 的位置不同和受刏割影响物候期推迟的两个方面综 合作用的结果。燕麦具有基部的分生组织, 3 个时 期的刈割处理都使燕麦增加了总分菜数。其中生殖 期重度刈割后总分箵数最多, 不施肥条件下有 4.36 个, 施肥条件下 8.83 个, 但是有效分蓝数却最少分 别为 1.08 与 1.63 个, 与对照植株相比分别减少 0.50 和 1.87 个。说明 3 个时期刚割处理促进了休 眠芽的活动, 且抽穗期达到最大。但是由于物候期 的延迟, 外界条件已不能满足其进一步再生生长的 需要。因此, 这时燕麦的补偿以营养补偿为主, 生殖 生长的补偿程度较小。不施肥条件下, 油菜营养期 的轻、重刚割处理后, 总分枝数分别为 1.63 和 1.54 个(对照植株为 1.78 个); 施肥条件下, 总分枝数分 别为 4.93 和 5.42 个 (对照植株为 6.61 个), 即营养 期的刈割对其生长有抑制作用, 生殖期的刈割均增 加了总分枝数。这和油菜休眠芽处于植株上部有 关, 只有经过一定时期的营养生长后休眠芽才出现。 即证明了我们的假设, 休眠芽位置以及活动造成植 物不同体型对补偿有很大的影响。

植物发生补偿反应的程度还与伤害的强度有关 (Maschinski \& Whitham, 1989; Bergelson \& Crawley, 1992; Lennartsson et al., 1998)。许多实验表明, 植 物在轻度伤害的情况下更易恢复补偿 (Obeso, 1998; Huhta et al., 2003)。本试验中轻度则割都有利于 燕麦、油菜的恢复补偿, 与该结论一致,即在低水平 的伤害情况下植物容易再生。燕麦和油菜轻度刈割 的条件下即可解除顶端优势, 刺激休眠芽的活动。 该情况不同于 Lennartsson 等( 1998)研究的一种假龙 胆属植物 (Gentianella campestris), 该植物在轻度刚割
（刚割部分占地上部分的 $10 \%$ ) 情况下没有超补偿， 重度刈割(刈割部分占地上部分的 50\%) 的情况下 反而出现了超补偿, 其原因可能是该植物需要较大 程度的刈割处理后顶端优势才能解除。

Hawkes 和 Sullivan (2001) 总结了前人的研究结 果表明, 大多数情况下禾草类植物在高资源的条件 下补偿效果好, 双子叶草本和木本植物的补偿与资 源的关系还不太明确。本文中燕麦在不施肥的条件 下, 补偿能力要好, 与陈红等 (2003) 所得结论一致。 油菜在施肥条件下补偿能力高, 其原因可能是燕麦 和油菜所经历的选择压力不同。燕麦作为高寒草甸 人工牧草, 自然选择使其投入到繁殖部分的能量较 低。不施肥条件下, 繁殖分配为 $(0.286 \pm 0.056)$, 施 肥条件下为 $(0.290 \pm 0.049)$, 方差分析不显著 $(d f=$ $119, p>0.05)$ 。而油菜作为油料作物, 人为选择使 其投入繁殖部分的能量较燕麦大。不施肥条件下为 $(0.392 \pm 0.088)$, 施肥条件下为 $(0.393 \pm 0.067)$, 方 差分析不显著 $(d f=119, p>0.05)$ 。就根冠比这个 指标来讲, 这两个物种对于施肥效应反应不一致。 施肥后油菜的根冠比为 $(0.130 \pm 0.043)$, 不施肥为 $(0.138 \pm 0.027)$, 方差分析不显著 $(d f=119, p>$ $0.05)$; 施肥后燕麦的根冠比为 $(0.077 \pm 0.024)$, 不 施肥为 $(0.097 \pm 0.063)$, 方差分析显著 $(d f=119, p$ $<0.05)$ 。施肥降低了燕麦的根冠比, 改变了植物生 长模式。刚割处理后影响了植物获取资源的能力。 因而施肥条件下补偿程度较低。当然对于该问题尚 需进一步的研究。

以生理和形态学为基础, 建立起来的植物忍耐 补偿机制, 在一定程度上能够解释植物的补偿反应。 但是还不具有一般性 (Tiffin, 2000)。许多试验的研 究只局限于一个物种或一个种群 (Caldwell et al.,

1981; van der Meijden et al., 1988; Rosenthal \& Welter, 1995)。比较不同物种或同一物种内不同补偿机 制的试验还较少见 ( Juenger \& Bergelson, 2000)。因 此, 本试验中我们还调查了燕麦、油菜新生叶片和残 留叶片的光合能力的比较, 新生叶片与残留叶片特 定叶面积干重 (SLA)、可溶性碳水化合物在根、茎、 叶、果实中的分配转移规律。定量分析 (通径分析) 的结果仍支持我们的假设 (结果另文发表)。

综上所述, 影响植物补偿反应的因素有多方面, 任何片面强调某个方面而发展起来的模型( Tuomi et al., 1994; Lehtilä, 2000)或理论( Hilbert et al., 1981; Maschinski \& Whitham, 1989; Weis et al., 2000)都不 可能具有普遍性。环境的异质性、季节性的差异、放 
牧史等也可影响植物补偿反应的表达。在这里我们 所说的植物补偿反应能力是指植物种群中所有个体 补偿反应能力的平均值。不同种群之间, 或者同一 种群内不同基因型的植物补偿反应能力可能不一样 (Martinez et al., 1999)。Paige 和 Whitham（1987）及 Bergelson 和 Crawley (1992) 研究同种材料 ( Ipomopsis aggregata) 的补偿反应不同, 可能就是该原因导致 的。这也从另一个方面证明植物的补偿反应形式不 具有一个统一的模式。

\section{参 考 文 献}

Belsky AJ (1986) . Does herbivory benefit plants? A review of the evidence. American Naturalist, 127, 870-892.

Belsky AJ, Carson WP, Jensen CL, Fox G (1993). Overcompensation by plants: herbivore optimization or red herring? Evolutionary Ecology, 7, 109 - 121 .

Bergelson J, Crawley MJ (1992). Herbivory and Ipomopsis aggregata: the disadvantages of being eaten. American Naturalist, $139,870-872$.

Caldwell MM, Richards JH, Johnson DA, Nowack RS, Dzurec RS (1981) . Coping with herbivory: photosynthetic capacity and resource allocation in two semiarid Agropyron bunchgrasses. Oecologia, 50,14-24.

Chen H(陈红), Wang HY (王海洋), DU GZ(杜国祯) (2003). Impacts of clipping time, clipping intensity and fertilization on plant compensation of Avena sativa. Acta Botanica Boreali-occidentalia Sinica (西北植物学报) , 23,969-975. (in Chinese with English abstract)

DU GZ (杜国祯), Wang G (王刚) (1995). Succession and changes of grassland quality of the artificial grassland communities in subalpine meadow in Gannan. Acta Botanica Sinica (植物学 报), 37,306-313. (in Chinese with English abstract)

Evans AS (1991). Whole-plant responses of Brassica campestris to altered sink-source relations. American Journal of Botany, 78, $394-400$.

Hawkes CV, Sullivan JJ(2001) . The impact of herb ivory on plants in different resource conditions: a meta-analysis. Ecology, 82, $2045-2058$.

Hilbert DW, Swift DM, Delting JK, Dyer MI (1981). Relative growth rates and the grazing optimization hypothesis. Oecologia, $51,14-48$.

Huhta AP, Hellström K, Rautio P, Tuomi J(2003) . Grazing tolerance of Gentianella amarelle and other monocarpic herbs: why is tolerance highest at low damage levels? Plant Ecology, 166,4961 .

Juenger T, Bergelson J (2000). The evolution of compensation to herbivory in scarlet gilia, Ipomopsis aggregata: herbivore-imposed natural selection and quantitative genetics of tolerance. Evolution, 54, $764-777$.

Lehtilä K (2000) . Modelling compensatory regrowth with bud dormancy and gradual activation of buds. Evolutionary Ecology, 14, $315-330$.

Lennartsson T, Tuomi J, Nilsson P (1998). Induction of overcompensation in the field gentian, Gentianella campestris. Ecology, $79,1061-1072$.

Martínez Moreno D, Núňez-Farfán $J$, Terrazas T, Ruiz LM, Trinidad-Santos A, Trejo C, Larque-Saabedra A (1999). Plastic responses to clipping in two species of Amaranthus from the Sierra Norte de Puebla, Mexico. Genetic Resources and Crop Evolution, $46,225-234$.

Maschinski J, Whitham TG (1989). The continuum of plant responses to herb ivory: the influence of plant association, nutrient availability, and timing. American Naturalist, 134,1-19.

Obeso JR (1998). Effects of the defoliation and girdling on fruit production in Ilex aquiafolium. Functional Ecology, 12, 486 491.

Paige KN, Whitham TG (1987). Overcompensation in response to mammalian herbivory: the advantage of being eaten. American Naturalist, 129,407-416.

Rosenthal JP, Welter SC(1995). Tolerance to herbivory by a stemboring caterpillar in architecturally distinct maizes and wild relatives. Oecologia, 102, 146 - 155 .

Rosenthal JP, Kotanen PM (1994). Terrestrial plant tolerance to herbivory. Trends in Ecology and Evolution, 9,145-148.

Tiffin $\mathrm{P}(2000)$. Mechanisms of tolerance to herbivore damage: what do we know? Evolutionary Ecology, 14,523 - 536 .

Trumble JT, Kolodny-Hirsch DM, Ting IP (1993) . Plant compensation for arthropod herbivory. Annual Review of Entomology, $38,93-119$.

Tuomi J, Nilsson P, Åström M (1994). Plant compensatory responses: bud dormancy as an adaptation to herbivory. Ecology, $75,1429-1436$.

van der Meijden E, Wijin M, Verkaar HJ(1988). Denfense and regrowth, alternative plant strategies in the struggle against herbivores. Oikos, 51, 355- 363.

Wang HY (王海洋), Du GZ (杜国祯), Ren QJ (任青吉) (2003) . The impacts of population density and fertilization on compensatory responses of Elymus nutans to mowing. Acta phytoecologica Sinica (植物生态学报) ,27, 477 - 483. (in Chinese with English abstract)

Weis AE, Simms EL, Hochberg ME(2000). Will plant vigor and tolerance be genetically correlated? Effects of intrinsic growth rate and self-limitation on regrowth. Evolutionary Ecology, 14,331 352 . 